\title{
Research on the Video Production Methods of Micro-lessons -- A Study Based on Computer Technology
}

\author{
Shuang Zhang ${ }^{1, a}$ \\ ${ }^{1}$ School of Physical Education, Wuhan Business University, Wuhan City, Hubei Province, China \\ atiredhiker@hotmail.com
}

Key words: micro-lessons, video production methods

\begin{abstract}
By using the methods of literature review and practical operation, we analyzed the video production methods of micro-lessons. The results showed that the production of micro-lessons mainly includes material acquiring, beginning and ending production and post editing. Video recording can be captured by recording or some software, camtasia studio, bandicam, fraps, Hanwei Micro-lesson, Screen Recorder, HyperCam, Corel Video Studio Pro and other software. The animation production of the videos can be achieved by Anime studio pro, flash, comic studio 3Dmax, Maya, SolidWorks, SoftImage and other software. The acquisition and production of audio material can adopt Cool Edit Pro GoldWave, ApowerSoft, Messer audio recording software, Sound Recorder and other software. Post-editing can adopt Corel Video Studio Pro, Editing Master, Premiere and Sound Effect Maker, etc.
\end{abstract}

\section{Introduction}

Currently, micro-lesson is a new worldwide teaching mode. Its essence is to present the knowledge to the learners with videos whose length is usually from five minutes to ten minutes. The length of the videos is short. Therefore, the learners can learn in fragmented time. At present, many schools add micro-lessons in the traditional teaching, which can not only improve the students' interest in learning, but also help the students make full use of their spare time. With micro-lessons, the students can learn at any time and any place. And micro-lessons can be spread in modern communication software, such as WeChat. This is a new kind of learning mode, as well as the further integration of the open universities and the society. There are several forms of producing micro-lessons. Firstly, we can outsource. The makers only need to provide their idea and the knowledge within the micro-lessons. Then they can teach the students in accordance with the designed mode. The service companies will shoot and clip the videos. Because of their higher technical levels, the micro-lessons are better and can save the teachers' time. But the cost of this kind of outsourcing is high. If the funds is enough, we can use this method. Secondly, the teachers can produce the videos with their own spare time, which requires the teachers' certain knowledge of computer technology, multimedia, film and video post processing. In the long run, this method can save cost and it is more conducive to the teachers to produce micro-lessons which are closely combined with knowledge. However, the teachers must master relevant knowledge and skills. For the teachers with large workload and little spare time, it is really difficult. In the present paper, the second method is mainly discussed. With the introduction of the current commonly used micro-lessons production software, the teachers will be able to have basic understanding of the production processes of micro-lessons and master the basic production methods. 


\section{Methods for Acquiring Raw Video Materials}

There are a variety of ways acquiring video material. The specific choice depends on the content of the micro-lessons and the teachers' design. Before getting the video raw material, we should make adequate preparations according to the design of the micro-lessons. If we acquire the original material by shooting, we need to prepare scenes, props, micro-lessons' shooting scripts. If we produce the videos by computer, we need to prepare pictures, animation, audio in accordance with the design.

Shooting. Shooting is the most direct and the most commonly used method to capture video material. In the actual operation, we can use a professional camera to shoot. If the micro-lessons do not have high requirements of the video elements, we can use the phone to shoot. Before video shooting, we can choose a good seat. We had better set a few seats to get rich raw material for post video clipping. In the process of shooting, if some parts are not clear enough or there is a typo, repeated fragments, or pet phrase, we can shoot it for many times. The preparations are very complicated. Thus, a shooting will consume a lot of manpower and material resources. In the process of shooting, we must grasp the chance to shoot more and acquire more material in order to provide sufficient raw materials for the video production.

Screen recording. Some typical courses, such as Computer Operation Demonstration, some theoretical knowledge, can use the method of screen recording to complete the production of micro-lesson video. In comparison, screen recording does not require complicated organization. The maker only needs a computer and network, then the video will be completed. But the screen recording mode requires that the maker should master some computer technologies. There are some commonly used software for screen recording, such as, camtasia studio, bandicam, fraps, Hanwei Micro-lesson, Screen Recorder, HyperCam, Corel Video Studio Pro, etc. The micro-lesson producers only need to master one of the above software. Then they can complete the screen recording. Camtasia studio is recommended. And its interface is shown in Figure 1.

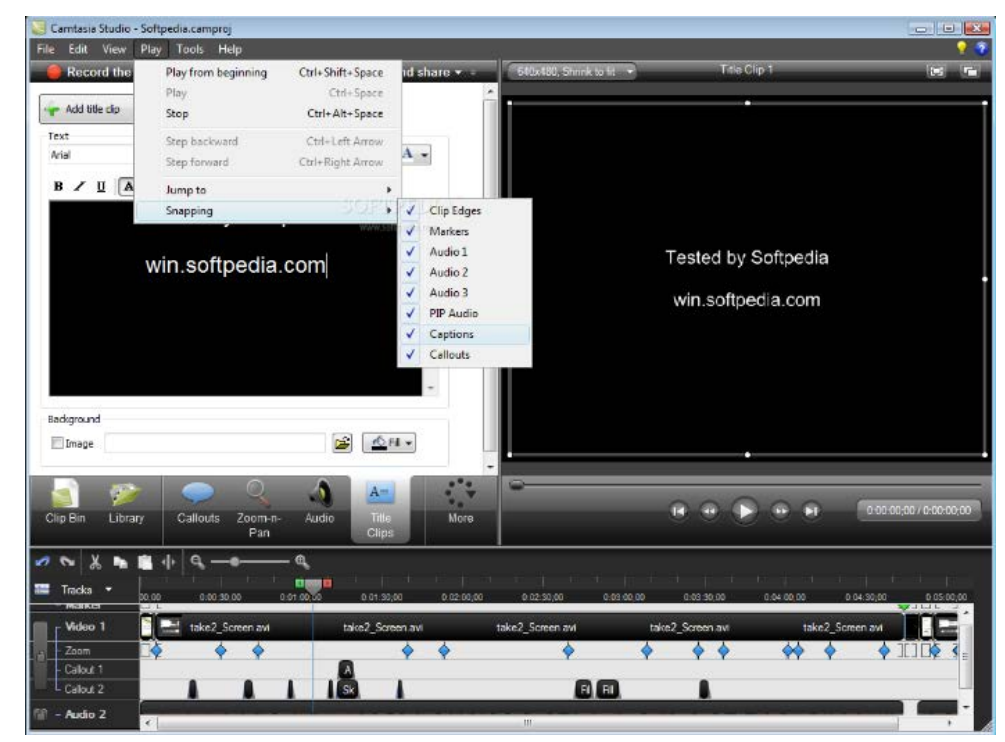

Figure 1 Camtasia studio interface

Animation production. Some knowledge can be presented in the form of animation. Animation is divided into 2D animation and 3D animation. And 2D animation production is easier than that of 3D animation.

The commonly used software of 2D animation are Anime studio pro, flash and comic studio, etc. Flash software is currently more popularly used and its usage is relatively simple. The users just need to be familiar with the basic operations of this software, then they can produce a perfect 2D 
animation. The flash interface is shown in Figure 2.

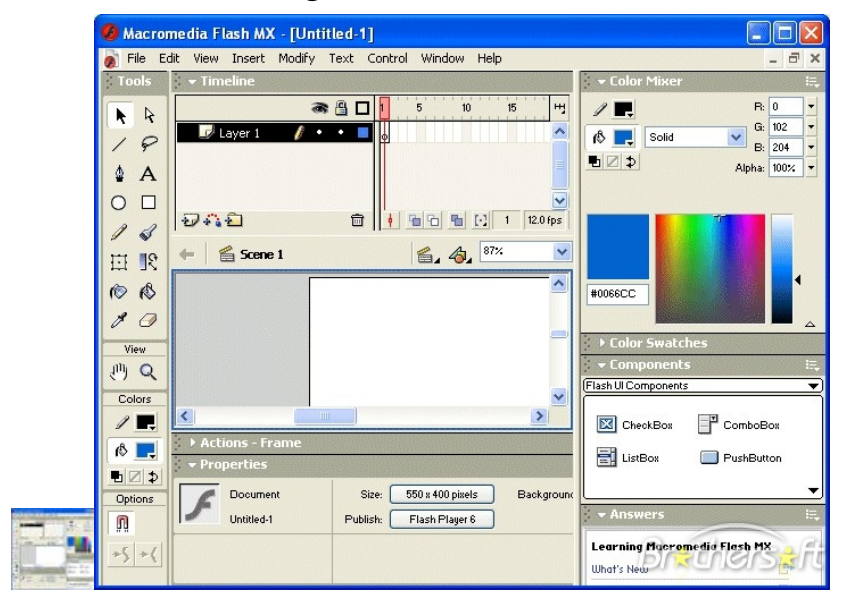

Figure 2 Flash interface

The commonly used software of 3D animation are 3Dmax, Maya, SolidWorks and SoftImage, etc. Among them, 3Dmax and Maya are widely used in China. The users need to spend a lot of time to master the basic operations of 3D software. However, the principles of these software are similar to each other. Since the user can operate a kind of the software, they will clearly operate the others. If the micro-lesson maker has plenty of time, he or she can learn the operation of 3Dmax. And 3Dmax interface is shown in Figure 3.

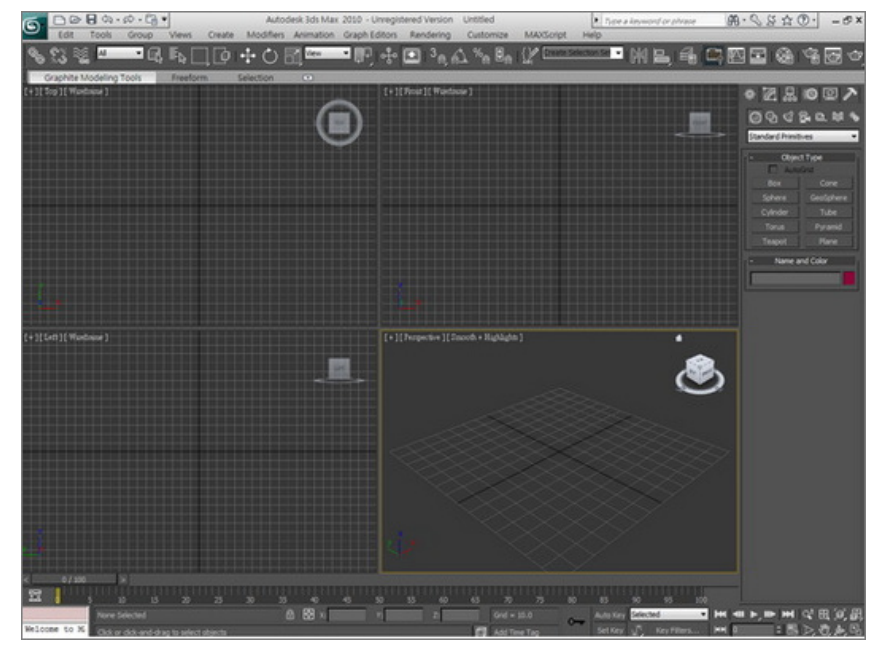

Figure 3 3Dmax interface

Whether it is 2D animation or 3D animation, it requires certain art foundation. The user should understand the basic principles of animation. If the user lacks the basic ability, it will be difficult to produce a beautiful animation. If the maker does not have the basic skills of art, it is recommended that he or she should make the micro-lessons with others' cooperation or outsourcing.

Acquiring photos. We can acquire photos by using professional SLR cameras, or smart phones. When taking pictures, we should pay attention to the clean and tidy shooting environment. In the environment with poor light, we can use to acquire the best shooting effect. The photographs can be later modified by Meituxiuxiu, a kind of software with simple operation, or Photoshop, a kind of professional software. Meituxiuxiu's operation is relatively simple, but its functions are limited. Photoshop can deeply process the photos and can get different visual effects. But its usage is relatively complex. Photoshop interface is shown in Figure 4. 


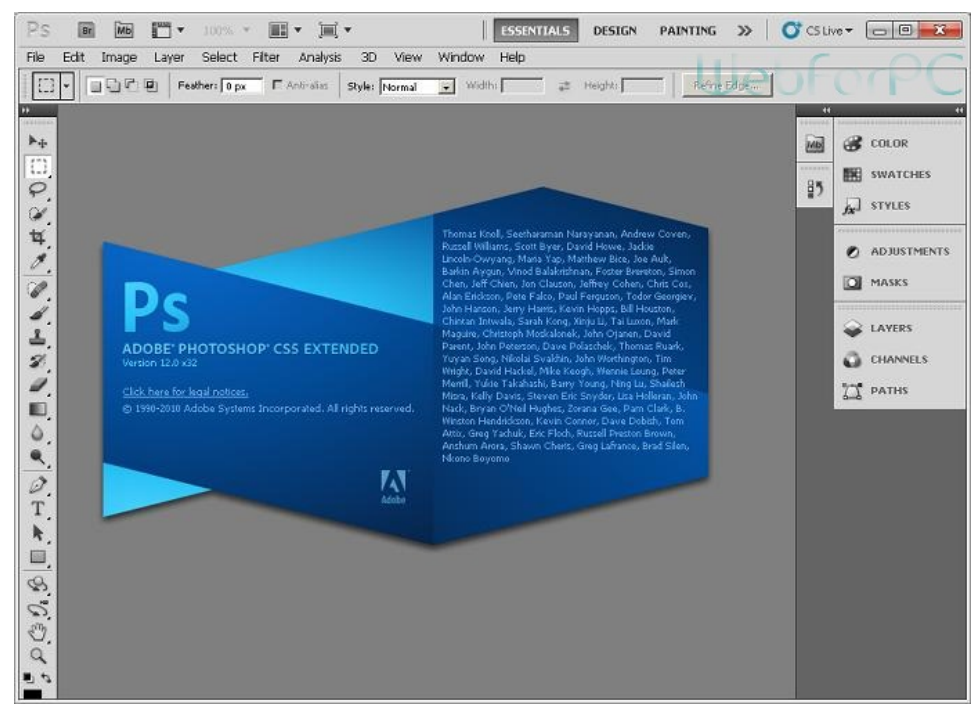

Figure 4 Photoshop interface

Acquiring audio resources. The first way to acquire audio resources is to download from the network. But the problem of copyright may occur and it is illegal to download these audio materials. We can not infringe upon the intellectual property rights of others. The other way is to use the method of recording to acquire the audio material. In the recording process, we need to ensure that the environment is quiet without noise.

There are many kinds of software for recording audio material. Firstly, we can use the computer's recording software. The software is easy to operate. The maker only needs to insert the microphone. Secondly, we can use the professional recording software for recording. The commonly used software for recording and audio processing is Cool Edit Pro, GoldWave, ApowerSoft, Messer Recording, Recording Master and so on. Thirdly, we can use the smart phone comes with recording software to record, or download the audio recording applications of smart phone for recording.

\section{The Production of the Beginning of the Video}

A complete video contains three parts, the beginning, the basic content and the ending. The beginning can adopt the form of simple text, or the complex text with animation effects. The ending generally adopts the text introduction, mainly including the workers' names handling photography, clipping, planning and the information of copyright.

The special effects of text. Static text with special effects can adopt Photoshop which is a professional photo processing software. It can produce a variety of static text with special effects. Dynamic text with special effects can adopt Xara 3D Maker, Ulead Cool 3D, SWFtext, etc. Among them, Ulead Cool 3D and SWFtext have simple operation and it is recommended to use.

The special effects of animation. The software making animation effects include After Effects, Houdini, Illusion, Fusion, Shake, etc. Among them, After Effects and Houdini are widely used. The tutorial videos on the network are quite rich. This software is recommended for learning. After Effects interface is shown in Figure 5. 


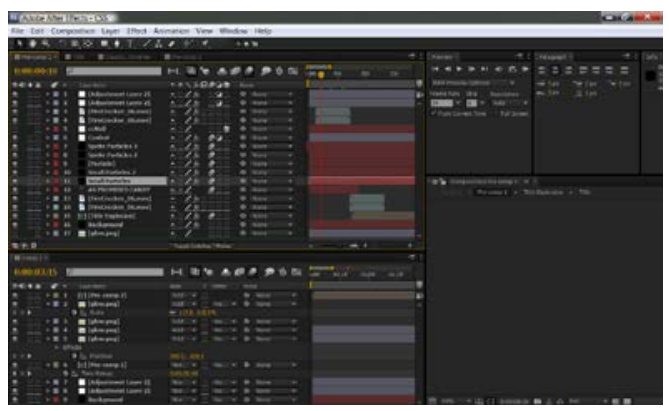

Figure 5 After Effects interface

\section{Video Clipping}

Video editing is the key to produce micro-lesson videos. During the processes of video clipping, the beginning and the ending of the video will be added. The materials with poor visual effect will be removed while those with good visual effect will be put together. The consistency of the video is also important. In addition, in the video clipping software can also handle audio materials. The schedule of the video and audio should be consistent. If it is specially recorded, the audio should be embedded into the video. Meanwhile, the synchronization of audio and video motion should be ensured.

The commonly used video clipping software include Editing Master, Corel Video Studio Pro, Premiere, etc. The Editing Master's functions are limited and its usage is inconvenient. Corel Video Studio Pro has perfect functions, but its operation is slightly more complicated. Premiere is a professional nonlinear video and audio editing software. Its operation is complex, powerful and it can produce a variety of video effects. These three commonly used software has their own characteristics. Premiere is recommended. Through this software, we can produce a video with perfect visual effect. The premiere interface is shown in Figure 6.

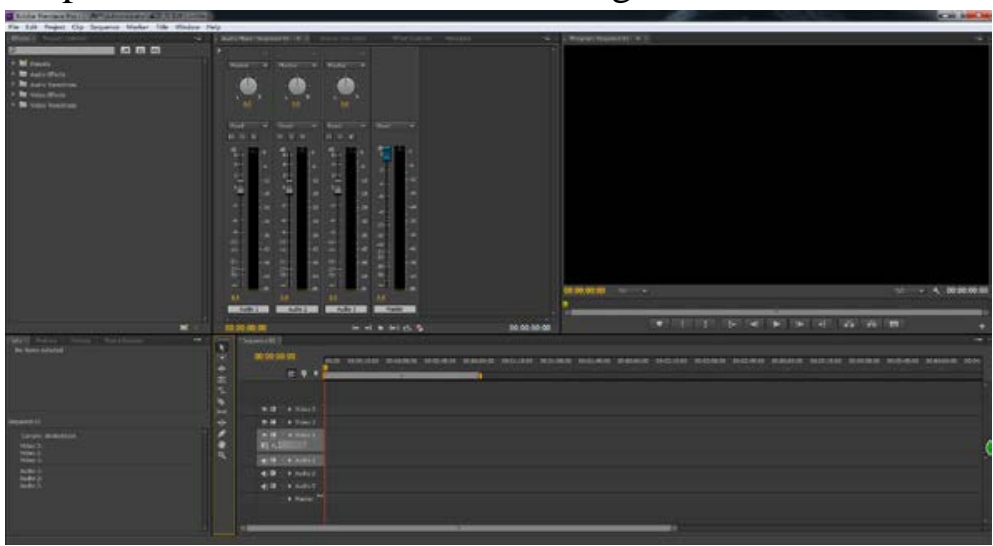

Figure 6 premiere interface

\section{Conclusions and Suggestions}

Conclusions. The production of micro-lessons can be based on the existing conditions and made by the producers themselves, which can save much money and make the effects of micro-lessons videos and knowledge more closely.

A complete micro-lesson is divided into three parts, namely, beginning, content and ending. Each part has the original material acquisition, the relevant production methods and software.

During the production processes of micro-lessons, we should make the best use of the existing conditions to achieve the best results. The planning and material preparations of micro-lessons is 
the basic guarantee for the completion of the project, which should be put into enough time and energy.

Suggestions. During the production of micro-lessons, the teachers should make the best use of the existing resources, devote enough energy and time in using the software and fully grasp the usage of various software to make the micro-lessons.

In the video processing, the hardware should be as perfect as possible, especially in the post video processing. The best computer equipped with high configuration is required. If the computer configuration is low, it will be a waste of time. This kind of time cost is a huge loss.

If there is no practice in the production of micro-lessons, the producers should learn from others with the internet. Thus, they could save time while producing the micro-lessons.

\section{References}

[1] Min Gu. Micro-lessons Making Learning Experience in Higher Education [J]. Software Engineer, 2014(9).

[2] Yong Peng. Discussion on higher vocational teaching model based on micro-learning resources [J]. Software Guide, 2015 (4).

[3] Bing-shan Yin. Application of micro-learning resources and MOOC in adult education [J]. Journal of Beijing Xuanwu Hongqi Spare-time University, 2015 (1).

[4] Xiao-ling Zhang. Application of micro-learning resources and MOOC in medical imaging teaching [J]. China Medical Herald, 2016 (3).

[5] Xiao-bing Su. Research on the Concept and Instructional Application of Micro-lessons [J]. China Educational Technology, 2014 (7).

[6] Yi-an Li \& Ying Li. Characteristics and strategy training of English learning in the environment of micro-lessons [J]Journal of Jilin Province Economic Management Cadre College, 2015 (2).

[7] Yi Fang, et al. Exploration of design in physical micro-lessons based on MOOC [J]. Physics and Engineering, 2014 (3). 